\section{Aconselhamento para a prática de atividade física como estratégia de educação à saúde}

\author{
Counseling for physical activity as a health \\ education strategy
}

\footnotetext{
1 Centro de Desportos, Universidade Federal de Santa Catarina, Florianópolis, Brasil. 2 Centro de Ciência da Vida e da Saúde, Universidade Católica de Pelotas, Pelotas, Brasil.

3 Faculdade de Medicina, Universidade Federal de Pelotas, Pelotas, Brasil. 4 Centro de Ciências Humanas e da Educação, Universidade Católica de Pelotas, Pelotas, Brasil. 5 Faculdade de Enfermagem e Obstetrícia, Universidade Federal de Pelotas, Pelotas, Brasil.

${ }^{6}$ Escola Superior de Educação Física Universidade Federal de Pelotas, Pelotas, Brasil.

Correspondência F. V. Siqueira

Programa de Pós-graduação em Educação Física, Centro de Desportos, Universidade Federal de Santa Catarina. C. P. 476, Campus Universitário Trindade, Florianópolis, SC 88040-900, Brasil. fcvsiqueira@uol.com.br
}

\begin{abstract}
This study analyzes the prevalence of health counseling related to physical activity among users of primary health care, and explores the associated variables. This cross-sectional study included a random sample of 4,060 adults and 4,003 older adults living in areas covered by primary health care in 41 cities from seven States of South and Northeast Brazil. Prevalence of counseling for physical activity was 28.9\% (95\%CI: 27.3-30.4) for adults and 38.9\% (95\%CI: 37.3-40.6) for elders. Prevalence was consistently higher among older adults, in the Northeast, and in areas covered by the Family Health Program. Gender, socioeconomic status, smoking, sedentary lifestyle, self-reported chronic non-communicable diseases, and continuous-use medication were also associated with the outcome. Counseling for physical activity at community health centers is under-promoted, considering individual needs for healthy living. There is a need to improve participation by health professionals from different fields to promote physical activity and other healthy behaviors.
\end{abstract}

Motor Activity; Counseling; Adult; Aged

\author{
Fernando Vinholes Siqueira 1,2 \\ Markus Vinícius Nahas 1 \\ Luiz Augusto Facchini 3 \\ Denise Silva Silveira ${ }^{3}$ \\ Roberto Xavier Piccini 3 \\ Elaine Tomasi 4 \\ Elaine Thumé 5 \\ Pedro Curi Hallal 6
}

\section{Introdução}

A Declaração de Alma-Ata em 1978 identificou as atividades de atenção primária à saúde que oportunizariam alcançar a meta de "Saúde para Todos" no ano 2000. Dentre essas atividades, destaca-se a educação da população sobre problemas prevalentes e seus métodos de prevenção e controle. Às vésperas de completar trinta anos, a Declaração se mantém atualizada quanto à necessidade de se buscar a prevenção e promoção à saúde de populações, com um crescente reconhecimento científico da relevância da atividade física como estratégia para este fim, inclusive com a identificação de pautas relativas à necessidade de mudança de comportamento, fundamentais para a promoção da saúde e prevenção de doenças.

Incorporar novas práticas saudáveis que possam trazer benefícios à população é um dos desafios impostos a toda a estrutura da atenção básica. Mudanças que sejam capazes de se transformar em práticas educativas para a saúde das populações devem ser realizadas a partir de um diálogo com os atores responsáveis pelas diferentes dimensões da atenção básica 1. Embora na atenção primária à saúde as ações "curativas" (diagnóstico e tratamento) ocupem boa parte do tempo dos profissionais de unidades básicas de saúde, sua participação em atividades educativas, como por exemplo, orientar a realização de atividade física, é fundamental para a difusão de 
comportamentos saudáveis para a população da área de cobertura dos serviços.

A atenção à saúde tem sido construída com base em práticas estabelecidas entre gestores, profissionais e população no sentido de atender às exigências impostas pelos usuários dos serviços de saúde. Espera-se assim, que a utilização de procedimentos em educação à saúde seja uma das estratégias que possibilite as mudanças necessárias no sentido de redirecionar gestão, práticas profissionais, de controle social e de estímulo à busca de comportamentos saudáveis por parte de todos que a utilizam 1,2 .

Entre essas necessidades está a aceitação de procedimentos relacionados ao aconselhamento e, entre eles, o aconselhamento à prática de atividade física nos atendimentos em saúde por parte dos profissionais que trabalham em unidades básicas de saúde. Alguns estudos têm demonstrado a importância da participação do profissional de saúde no sentido de educar e estimular mudanças de comportamento nas pessoas. Entretanto, no Brasil, ainda é escasso o conhecimento sobre a orientação de atividade física como estratégia de educação à saúde na rede básica 3,4. Essa importância fica fortalecida com as conclusões de Borjesson \& Dahlof 5, referindo que a atividade física é muito pouco utilizada no cuidado à saúde, na prevenção e no tratamento, principalmente de algumas morbidades como a hipertensão, em que o efeito da atividade física na redução da pressão arterial é similar ao da terapia farmacológica.

O presente artigo contribui para o preenchimento da lacuna na literatura sobre a atividade física como estratégia de educação em saúde na rede básica, e tem como objetivo descrever a prevalência de aconselhamento educativo à saúde relacionado à atividade física, entre as pessoas que utilizaram alguma vez na vida uma unidade básica de saúde. O estudo também verifica alguns fatores associados com o aconselhamento para realização de atividade física.

\section{Metodologia}

Foi realizado um estudo de delineamento transversal com uma amostra de indivíduos adultos (30 a 64 anos) e idosos (65 anos ou mais), residentes nas áreas de abrangência das unidades básicas de saúde de 41 municípios com mais de 100 mil habitantes dos estados do Rio Grande do Sul e Santa Catarina, na Região Sul; e Alagoas, Pernambuco, Paraíba, Rio Grande do Norte e Piauí na Região Nordeste do Brasil 6 .

Os municípios incluídos no estudo compõem os Lotes 2 - Sul e Nordeste - do Estudo de
Linha de Base do Projeto de Expansão e Consolidação do Saúde da Família (PROESF) 2. Uma amostra aleatória de 120 unidades básicas de saúde foi sorteada em cada um dos lotes estudados, com distintas modalidades de atenção básica - tradicional e Programa Saúde da Família (PSF). Unidades básicas tradicionais foram consideradas aquelas que se caracterizavam pela presença na equipe de médicos especialistas (clínico, pediatra e gineco-obstetra), enfermeiro, auxiliar de enfermagem e pessoal administrativo para apoio técnico. Muitas dessas unidades contam com o apoio esporádico de outras especialidades profissionais (dentista, nutricionista, fisioterapeuta) e mesmo médicas. A população atendida caracteriza-se por ser espontânea ou devido a encaminhamento de outros serviços. As unidades de PSF, ao contrário, são formadas por equipes de saúde da família, que incluem médico generalista, enfermeiro, auxiliares de enfermagem e agentes comunitários, responsáveis pelo cadastramento e acompanhamento da população residente na área de abrangência do serviço 7 .

Baseando-se em listas produzidas pelos municípios, as unidades básicas de saúde foram selecionadas de forma aleatória, respeitando a proporcionalidade por modelo de atenção. A seleção da amostra nos municípios foi proporcional à capacidade instalada de sua rede básica. $\mathrm{Na}$ Região Sul, obteve-se uma amostra de 69 unidades básicas de saúde de PSF e 51 tradicionais. No Nordeste, a amostra foi constituída de 79 unidades básicas de saúde de PSF e 41 tradicionais.

Após a amostragem, as unidades básicas de saúde sorteadas orientaram a seleção da amostra, que foi realizada de forma sistemática e independente para a seleção de adultos e idosos. A amostra foi estimada em 2.100 indivíduos por lote (4.200 para o Sul e o Nordeste), de modo a alcançar poder estatístico de, no mínimo $80 \%$, para eventos de prevalência aproximada de $25 \%$. O número estimado de indivíduos para cada lote (2.100) foi dividido pelo número total de unidades básicas de saúde (120 unidades/lote), obtendo-se a necessidade de entrevistar 18 adultos e 18 idosos na área de abrangência de cada uma das unidades básicas de saúde 6 .

A estratégia para delimitação da área de abrangência da unidade básica de saúde incluiu a obtenção prévia de seu mapa e uma estimativa populacional com base nas áreas censitárias do Instituto Brasileiro de Geografia e Estatística (IBGE; http://www.ibge.gov.br). A localização dos indivíduos na área delimitada ocorreu por meio de amostragem sistemática e incluiu apenas um indivíduo por domicílio. A coleta de dados foi realizada usando-se um questionário aplicado 
por 15 supervisores de campo após período de treinamento.

A definição do desfecho utilizada para o estudo, aconselhamento educativo à saúde relacionado à atividade física na unidade básica de saúde, foi operacionalizada por meio da pergunta: "Alguma vez na vida, em uma consulta no [Posto de Saúde de Abrangência], algum médico lhe disse que o(a) Sr.(a) deveria fazer exercícios para melhorar a sua saúde?". O questionário utilizado no estudo foi padronizado e pré-testado.

As variáveis independentes incluídas nesta análise foram: (a) sexo; (b) idade; (c) cor da pele (classificada pelo entrevistador baseando-se em observação); (d) padrão de consumo sócio-econômico (classificação da Associação Brasileira de Empresas de Pesquisa - ABEP 8) ; (e) situação conjugal; (f) tabagismo; (g) sedentarismo (escore de atividade física inferior a 150 minutos por semana, obtido com base no Questionário Internacional de Atividade Física 9); (h) diagnóstico médico referido de hipertensão; (i) diagnóstico médico referido de diabetes; (j) diagnóstico médico referido de doença dos nervos; (k) número de medicamentos referidos para uso contínuo e; (l) autopercepção de saúde.

As análises descritivas incluíram cálculos de proporções e respectivos intervalos de 95\% de confiança (IC95\%). Na análise bruta, a prevalência de orientação para atividade física foi calculada para cada grupo das variáveis independentes, e o nível de significância foi testado usando-se os testes de Wald para heterogeneidade e tendência linear. A análise ajustada foi realizada por regressão de Poisson, com ajuste robusto de variância, com cálculo de razões de prevalência (RP) ajustadas, IC95\%, e valores de significância (p) usando-se os mesmos testes descritos acima. Todas as análises levaram em consideração o desenho amostral e foi utilizado um modelo hierárquico de determinação do desfecho 10. No primeiro nível de determinação foram analisadas as variáveis a, b, c, d. No segundo nível as variáveis e, f, g. No terceiro as variáveis $h, i, j$. No quarto nível as variáveis $k, l$. Todas as variáveis com $p \leq 0,20$ foram mantidas no modelo de regressão com a finalidade de promover controle para possíveis fatores de confusão. Toda a análise está ajustada por nível e para aquelas de níveis superiores na determinação do desfecho 10 .

Variáveis de contexto também foram utilizadas para descrever possíveis diferenças na orientação de atividade física. Para isso utilizamos as variáveis relacionadas aos estados, as regiões Sul e Nordeste e o modelo de atenção, PSF ou tradicional.

O Comitê de Ética da Faculdade de Medicina da Universidade Federal de Pelotas aprovou o protocolo do estudo, e foi obtido consentimento informado de todos os participantes.

\section{Resultados}

Do total de entrevistas nos estados da Região Sul, 1.940 foram realizadas com indivíduos adultos e 1.891 com idosos. Na Região Nordeste, 2.120 adultos e 2.112 idosos foram entrevistados. As taxas de não respostas foram de 3,5\% e 4,7\% para adultos e idosos, respectivamente.

A prevalência de aconselhamento educativo à saúde relacionado à atividade física entre os moradores que utilizaram alguma vez na vida uma unidade básica de saúde foi de 28,9\% (IC95\%: 27,3-30,4) entre os adultos e de 38,9\% (IC95\%: $37,3-40,6)$ entre os idosos. Essa prevalência entre os adultos do Sul foi de 26,6\% (IC95\%: 24,3-28,8) e do Nordeste $31 \%$ (IC95\%: 28,8-33,1). Entre os idosos as prevalências foram de 35,5\% (IC95\%: $33,3-38,0$ ) e $41,8 \%$ (IC95\%: 40,0-44,1) para o Sul e o Nordeste, respectivamente. A prevalência de aconselhamento educativo à saúde relacionado à atividade física foi sempre maior entre os idosos e na Região Nordeste.

A Figura 1 mostra as prevalências do desfecho segundo os estados incluídos no estudo. O Estado de Alagoas mostrou a maior prevalência de aconselhamento à prática de atividade física tanto para adultos, 42,5\% (IC95\%: 35,5-49,5) quanto para idosos, 51,5\% (IC95\%: 44,5-58,4). Houve uma freqüência estatisticamente maior de aconselhamento nos estados da Região Nordeste, para as duas categorias de idade $(\mathrm{p}<0,001)$.

A Tabela 1 descreve a prevalência do desfecho em relação a algumas variáveis de contexto, como o modelo de atenção à saúde e a região. Pode-se verificar que a orientação educativa à saúde relacionada à atividade física foi significativamente maior nas unidades do modelo PSF independente do grupo populacional, sendo que no grupo de idosos esta orientação foi maior que a encontrada no grupo de adultos, tanto na amostra geral como nas regiões Sul e Nordeste.

A Tabela 2 descreve a amostra de acordo com as variáveis independentes utilizadas no estudo. O grupo de mulheres é maior tanto para adultos quanto para os idosos. A média de idade entre os adultos foi de 45,5 anos (desvio-padrão $\mathrm{DP}=9,6)$ e entre os idosos 73,9 ( $\mathrm{DP}=7,0)$. A prevalência de indivíduos de cor de pele branca foi aproximadamente o dobro da de não-brancos, tanto para o grupo de adultos como para o de idosos. O padrão de consumo sócio-econômico entre os adultos indicou uma prevalência maior na categoria agrupada entre A, B e C $(37,4 \%)$, enquanto entre os idosos a maior prevalência foi 
Figura 1

Prevalência de aconselhamento educativo à saúde relacionado à prática de atividade física nas unidades básicas de saúde, para adultos e idosos segundo o estado.

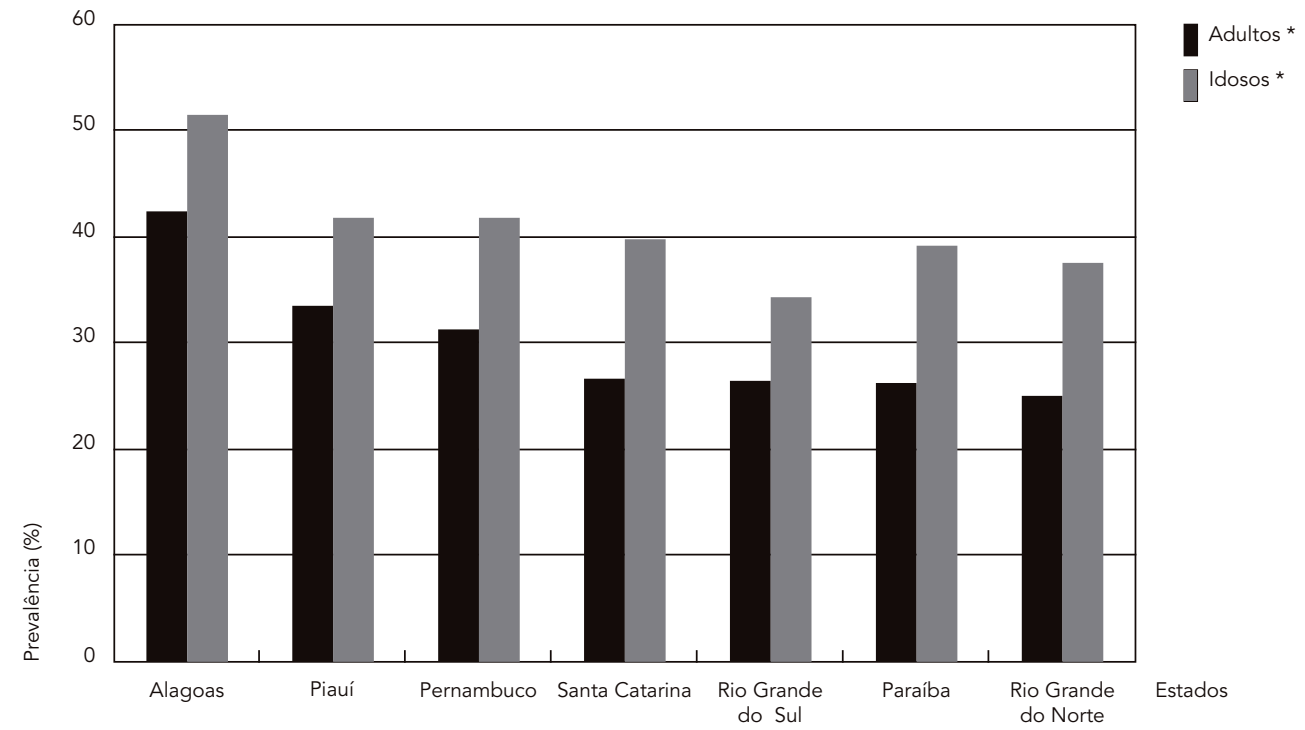

* Valor de $p<0,001$ pelo teste de Wald para heterogeneidade.

Tabela 1

Prevalência (IC95\%) de orientação para atividade física nas unidades básicas de saúde de acordo com o modelo de assistência e região, em áreas de abrangência de unidades básicas de saúde em municípios do Sul e Nordeste do Brasil.

\begin{tabular}{|c|c|c|c|c|c|c|c|c|}
\hline \multirow[t]{3}{*}{ Variável desfecho } & \multicolumn{4}{|c|}{ Programa Saúde da Família } & \multicolumn{4}{|c|}{ Tradicional } \\
\hline & \multicolumn{2}{|c|}{ Adultos } & \multicolumn{2}{|c|}{ Idosos } & \multicolumn{2}{|c|}{ Adultos } & \multicolumn{2}{|c|}{ Idosos } \\
\hline & $n$ & \% (IC95\%) & $\mathrm{n}$ & \% (IC95\%) & $\mathrm{n}$ & $\%$ (IC95\%) & $n$ & \% (IC95\%) \\
\hline \multicolumn{9}{|l|}{ Aconselhamento } \\
\hline \multicolumn{9}{|l|}{ educativo para } \\
\hline \multicolumn{9}{|l|}{ atividade física na } \\
\hline \multicolumn{9}{|l|}{ unidade básica de } \\
\hline \multicolumn{9}{|l|}{ saúde alguma vez } \\
\hline \multicolumn{9}{|l|}{ na vida } \\
\hline Toda amostra & 1.121 & $31,8(29,8-33,8)$ & 2.232 & $42,6(40,6-44,7)$ & 2.117 & $23,3(20,8-25,8)$ & 1.163 & $31,8(29,1-34,4)$ \\
\hline Região Sul & 899 & $28,7(25,7-31,7)$ & 947 & $38,1(35,0-41,2)$ & 630 & $23,5(20,2-26,8)$ & 658 & $32,1(28,5-35,6)$ \\
\hline Região Nordeste & 1.218 & $34,2(31,5-36,8)$ & 1.285 & $45,9(43,2-48,6)$ & 491 & $23,0(19,3-26,8)$ & 505 & $31,4(27,4-35,5)$ \\
\hline
\end{tabular}

Nota: todos os valores de $\mathrm{p}$ foram estatisticamente significativos $(<0,05)$ utilizando-se o teste de Wald para heterogeneidade.

na categoria E (45,3\%). A proporção de adultos que são casados ou vivem com companheiro(a) foi elevada $(73,3 \%)$, enquanto entre os idosos, os viúvos e os casados apresentaram proporções aproximadas. A prevalência de tabagismo foi de
$27,7 \%$ entre os adultos e de $15,2 \%$ nos idosos. O sedentarismo foi claramente superior entre os idosos (58\%) em relação aos adultos $(31,8 \%)$. Entre os grupos de adultos e idosos, os últimos mostraram consistentemente prevalências maiores 
Descrição das variáveis independentes em adultos e idosos moradores em áreas de abrangência de unidades básicas de saúde em municípios do Sul e Nordeste do Brasil.

\begin{tabular}{|c|c|c|}
\hline Variáveis & Adultos (\%) & Idosos (\%) \\
\hline \multicolumn{3}{|l|}{ Sexo } \\
\hline Masculino & 44,9 & 38,8 \\
\hline Feminino & 55,1 & 61,2 \\
\hline $\mathrm{N}$ & 4.060 & 4.003 \\
\hline \multicolumn{3}{|l|}{ Idade (anos) } \\
\hline $30-40$ & 36,2 & - \\
\hline $41-50$ & 31,1 & - \\
\hline $51-64$ & 32,7 & - \\
\hline $65-70$ & - & 38,5 \\
\hline $71-75$ & - & 25,7 \\
\hline $76-79$ & - & 18,5 \\
\hline 80 ou mais & - & 17,3 \\
\hline $\mathrm{N}$ & 4.059 & 4.003 \\
\hline \multicolumn{3}{|l|}{ Cor da pele } \\
\hline Branca & 65,3 & 70,0 \\
\hline Não-branca & 34,7 & 30,0 \\
\hline $\mathrm{N}$ & 4.039 & 3.985 \\
\hline \multicolumn{3}{|l|}{ Padrão de consumo sócio-econômico * } \\
\hline$A, B$ ou $C$ & 37,4 & 21,0 \\
\hline $\mathrm{D}$ & 32,4 & 33,7 \\
\hline$E$ & 30,2 & 45,3 \\
\hline $\mathrm{N}$ & 3.908 & 3.650 \\
\hline \multicolumn{3}{|l|}{ Situação conjugal } \\
\hline Casado(a) ou vive com companheiro(a) & 73,3 & 42,7 \\
\hline Viúvo(a) & 7,1 & 45,0 \\
\hline Separado(a)/Divorciado(a) & 9,8 & 7,8 \\
\hline Solteiro(a) & 9,8 & 4,5 \\
\hline $\mathrm{N}$ & 4.060 & 3.970 \\
\hline \multicolumn{3}{|l|}{ Tabagismo } \\
\hline Não, nunca fumou & 45,9 & 43,4 \\
\hline Já fumou & 26,4 & 41,4 \\
\hline Fumante & 27,7 & 15,2 \\
\hline $\mathrm{N}$ & 4.060 & 4.000 \\
\hline \multicolumn{3}{|l|}{ Sedentarismo } \\
\hline Não & 68,2 & 42,0 \\
\hline Sim & 31,8 & 58,0 \\
\hline $\mathrm{N}$ & 4.023 & 3.944 \\
\hline \multicolumn{3}{|c|}{ Diagnóstico médico referido de hipertensão arterial } \\
\hline Não & 72,0 & 36,5 \\
\hline Sim & 28,0 & 63,5 \\
\hline $\mathrm{N}$ & 3.960 & 3.963 \\
\hline \multicolumn{3}{|c|}{ Diagnóstico médico referido de diabetes mellitus } \\
\hline Não & 93,3 & 80,5 \\
\hline Sim & 6,7 & 19,5 \\
\hline $\mathrm{N}$ & 3.897 & 3.909 \\
\hline \multicolumn{3}{|c|}{ Diagnóstico médico referido de doença dos nervos } \\
\hline Não & 74,0 & 71,0 \\
\hline Sim & 26,0 & 29,0 \\
\hline $\mathrm{N}$ & 4.033 & 3.956 \\
\hline
\end{tabular}

(continua) 


\begin{tabular}{lcc} 
Tabela 2 (continuação) & \\
\hline Variáveis & Adultos (\%) & Idosos (\%) \\
\hline Número de medicamentos referidos para uso contínuo & & \\
$\quad$ Nenhum & 68,8 & 31,7 \\
1-2 & 22,0 & 39,0 \\
3 ou mais & 9,2 & 29,3 \\
N & 4.060 & 4.003 \\
Autopercepção da saúde & & \\
Excelente & 6,7 & 2,6 \\
Muito boa & 6,3 & 3,2 \\
Boa & 38,3 & 29,7 \\
Regular & 38,2 & 43,8 \\
Ruim & 10,5 & 20,7 \\
N & 4.048 & 3.951 \\
\hline
\end{tabular}

* De acordo com a classificação da Associação Brasileira de Empresas de Pesquisa (ABEP) 8.

de diagnóstico médico referido de hipertensão arterial, diabetes e doença dos nervos. Cerca de $2 / 3$ da população de adultos $(68,8 \%)$ referiram não fazer uso regular de nenhum medicamento, enquanto entre os idosos esta prevalência foi de $31,7 \%$. Tanto para os grupos de adultos quanto de idosos, as categorias de autopercepção de saúde boa e regular foram as de maior prevalência.

Os resultados da associação bruta e ajustada entre as variáveis independentes e o desfecho "aconselhamento educativo para a prática de atividade física recebido na unidade básica de saúde", estão apresentados na Tabela 3 . O aconselhamento para a prática de atividade física esteve fortemente associado às mulheres no grupo de adultos, tanto na análise bruta como na ajustada ( $R P=1,88$; IC95\%: 1,66-2,13). Entre as idosas, o desfecho mostrou associação somente na análise ajustada, porém de menor magnitude $(\mathrm{RP}=1,13$; IC95\%: 1,03-1,25). No grupo de adultos, a orientação educativa à saúde relacionada à atividade física tendeu a crescer com o aumento da idade ( $p<0,001)$. Uma tendência inversa é observada para o grupo de idosos; as menores chances de recebimento de aconselhamento ocorrem nos idosos de 80 anos ou mais $(\mathrm{RP}=0,68$; IC95\%: $0,59-0,80$ )

A associação entre o desfecho e o padrão de consumo sócio-econômico entre adultos perdeu magnitude na análise ajustada, embora tenha mantido significância estatística. A situação conjugal mostrou-se associada com aconselhamento à saúde, sendo que os que menos receberam aconselhamento nas unidades básicas de saúde foram os solteiros. Fumantes atuais apresentaram menor probabilidade de receberem aconselhamento à prática de atividade física, especialmente entre os idosos.
O sedentarismo esteve associado com aconselhamento para atividade física somente entre os adultos. Referir diagnóstico médico para hipertensão, diabetes e doença dos nervos esteve associado com aconselhamento educativo para atividade física nos dois grupos estudados na análise bruta. No grupo de idosos, na análise ajustada, o diagnóstico médico de doença dos nervos perdeu a significância estatística. Existe uma clara tendência para os indivíduos adultos e idosos de que usar três ou mais medicamentos de uso contínuo esteja associado com o aconselhamento educativo à saúde para prática de atividade física. Observou-se também uma associação entre aconselhamento para a prática de atividade física e percepção de saúde ruim, porém somente para o grupo de adultos.

\section{Discussão}

As prevalências de aconselhamento à prática de atividade física nas unidades básicas de saúde mostraram-se baixas, tanto para o grupo de adultos quanto para o de idosos, independente da região ou do modelo de assistência, especialmente se considerarmos que as unidades básicas de saúde devem ser porta de entrada do sistema de saúde e, portanto, nestes locais deveria existir uma ação integrada entre as diversas áreas de conhecimento da saúde, no sentido de beneficiar a população e promover mudanças significativas em direção a um estilo de vida saudável. Nosso achado sobre a orientação para atividade física é inferior ao observado nos Estados Unidos por Eden et al. 11 , que mostraram que $42 \%$ dos adultos que consultaram em uma unidade de cuidados primários à saúde foram aconselha- 
Prevalência de aconselhamento para atividade física, análises bruta e ajustada, para adultos e idosos moradores em áreas de abrangência de unidades básicas de saúde de estados do Sul e Nordeste do Brasil.

\begin{tabular}{|c|c|c|c|c|c|c|c|c|c|c|}
\hline \multirow[t]{3}{*}{ Variáveis [nível] } & \multicolumn{6}{|c|}{ Adultos } & \multicolumn{4}{|c|}{ Idosos } \\
\hline & \multicolumn{3}{|c|}{ Análise bruta } & \multicolumn{3}{|c|}{ Análise ajustada } & \multicolumn{2}{|l|}{ Análise bruta } & \multicolumn{2}{|c|}{ Análise ajustada } \\
\hline & $\%$ & RP (IC95\%) & $p$ * & RP (IC95\%) & $p$ * & $\%$ & RP (IC95\%) & $p$ * & RP (IC95\%) & $p$ * \\
\hline Sexo [1] & & & $<0,001$ & & $<0,001$ & & & 0,16 & & 0,01 \\
\hline Masculino & 19,8 & 1,00 & & 1,00 & & 37,4 & 1,00 & & 1,00 & \\
\hline Feminino & 35,6 & $1,80(1,59-2,03)$ & & $1,88(1,66-2,13)$ & & 39,8 & $1,06(0,97-1,16)$ & & $1,13(1,03-1,25)$ & \\
\hline Idade & & & $<0,001 *$ & & $<0,001 * \star$ & & & $<0,001 * *$ & & $<0,001 * \star$ \\
\hline \multicolumn{11}{|l|}{ (anos) [1] } \\
\hline $30-40$ & 18,9 & 1,00 & & 1,00 & & - & - & & - & \\
\hline $41-50$ & 30,1 & $1,59(1,37-1,86)$ & & $1,59(1,36-1,86)$ & & - & - & & - & \\
\hline $51-64$ & 38,1 & $2,02(1,75-2,33)$ & & $2,09(1,81-2,42)$ & & - & - & & - & \\
\hline $65-70$ & - & - & & - & & 42 & 1,00 & & 1,00 & \\
\hline $71-75$ & - & - & & - & & 39,6 & $0,94(0,85-1,04)$ & & $0,93(0,84-1,04)$ & \\
\hline $76-79$ & - & - & & - & & 40 & $0,95(0,85-1,07)$ & & $0,96(0,85-1,08)$ & \\
\hline 80 ou mais & - & - & & - & & 29,5 & $0,70(0,61-0,81)$ & & $0,68(0,59-0,80)$ & \\
\hline Cor da pele [1] & & & 0,25 & & 0,4 & & & 0,88 & & 0,61 \\
\hline Branca & 28,1 & 1,00 & & 1,00 & & 38,8 & 1,00 & & 1,00 & \\
\hline Não-branca & 30,4 & $1,07(0,96-1,20)$ & & $1,05(0,94-1,18)$ & & 39,1 & $1,01(0,92-1,10)$ & & $0,98(0,88-1,07)$ & \\
\hline Padrão de consumo & & & $0,01 * \star$ & & $0,03 * \star$ & & & $0,17 * \star$ & & $0,05 * *$ \\
\hline \multicolumn{11}{|l|}{ 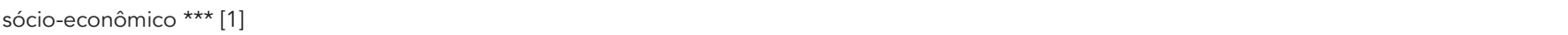 } \\
\hline$A, B$ ou $C$ & 26,1 & 1,00 & & 1,00 & & 35 & 1,00 & & 1,00 & \\
\hline $\mathrm{D}$ & 30,3 & $1,16(1,02-1,33)$ & & $1,12(0,98-1,28)$ & & 41,5 & $1,18(1,04-1,35)$ & & $1,19(1,05-1,36)$ & \\
\hline$E$ & 30,8 & $1,18(1,03-1,36)$ & & $1,15(1,01-1,31)$ & & 39,4 & $1,12(0,99-1,27)$ & & $1,16(1,03-1,32)$ & \\
\hline Situação conjugal [1] & & & $<0,001$ & & 0,01 & & & 0,01 & & 0,004 \\
\hline $\begin{array}{l}\text { Casado(a) ou vive } \\
\text { com companheiro(a) }\end{array}$ & 28,6 & 1,00 & & 1,00 & & 42 & 1,00 & & 1,00 & \\
\hline Viúvo(a) & 38,4 & $1,35(1,13-1,60)$ & & $0,93(0,78-1,11)$ & & 37,4 & $0,89(0,82-0,97)$ & & $0,87(078-097)$ & \\
\hline \multicolumn{11}{|l|}{ Separado(a)/ } \\
\hline Divorciado(a) & 33,5 & $1,17(1,00-1,39)$ & & $0,96(0,82-1,14)$ & & 34,2 & $0,82(0,68-0,97)$ & & $0,77(0,64-0,93)$ & \\
\hline Solteiro(a) & 19,1 & $0,67(0,53-0,84)$ & & $0,68(0,54-0,86)$ & & 34,4 & $0,82(0,66-1,02)$ & & $0,77(0,61-0,97)$ & \\
\hline Tabagismo [2] & & & $<0,001$ & & 0,03 & & & $<0,001$ & & $<0,001$ \\
\hline Não, nunca fumou & 29,2 & 1,00 & & 1,00 & & 39,3 & 1,00 & & 1,00 & \\
\hline Já fumou & 33,1 & $1,13(1,00-1,28)$ & & $1,09(0,96-1,23)$ & & 41,6 & $1,06(0,97-1,16)$ & & $1,08(0,98-1,19)$ & \\
\hline Fumante & 24,2 & $0,83(0,72-0,95)$ & & $0,88(0,77-1,02)$ & & 30,5 & $0,78(0,67-0,90)$ & & $0,79(0,68-0,93)$ & \\
\hline Sedentarismo [2] & & & 0,04 & & 0,02 & & & 0,5 & & 0,96 \\
\hline Não & 27,8 & 1,00 & & 1,00 & & 39,7 & 1,00 & & 1,00 & \\
\hline $\operatorname{Sim}$ & 31,4 & $1,13(1,01-1,26)$ & & $1,14(1,02-1,28)$ & & 38,5 & $0,97(0,89-1,06)$ & & $1,00(0,91-1,09)$ & \\
\hline Diagnóstico referido de & & & $<0,001$ & & $<0,001$ & & & $<0,001$ & & $<0,001$ \\
\hline \multicolumn{11}{|l|}{ hipertensão arterial [3] } \\
\hline Não & 20,8 & 1,00 & & 1,00 & & 25,2 & 1,00 & & 1,00 & \\
\hline $\operatorname{Sim}$ & 48,4 & $2,33(2,10-2,58)$ & & $1,80(1,65-2,09)$ & & 46,7 & $1,85(1,66-2,07)$ & & $1,73(1,54-1,94)$ & \\
\hline $\begin{array}{l}\text { Diagnóstico referido de } \\
\text { diabetes mellitus [3] }\end{array}$ & & & $<0,001$ & & $<0,001$ & & & $<0,001$ & & 0,02 \\
\hline Não & 27,8 & 1,00 & & 1,00 & & 36,7 & 1,00 & & 1,00 & \\
\hline $\operatorname{Sim}$ & 31,4 & $2,13(1,87-2,41)$ & & $1,60(1,40-1,84)$ & & 48,9 & $1,33(1,21-1,46)$ & & $1,18(1,07-1,30)$ & \\
\hline $\begin{array}{l}\text { Diagnóstico referido de } \\
\text { doença dos nervos [3] }\end{array}$ & & & $<0,001$ & & 0,02 & & & 0,02 & & 0,6 \\
\hline Não & 25,2 & 1,00 & & 1,00 & & 37,6 & 1,00 & & 1,00 & \\
\hline Sim & 38,4 & $1,53(1,37-1,70)$ & & $1,14(1,02-1,28)$ & & 41,9 & $1,11(1,02-1,22)$ & & $1,03(0,93-1,13)$ & \\
\hline
\end{tabular}

(continua) 
Tabela 3 (continuação)

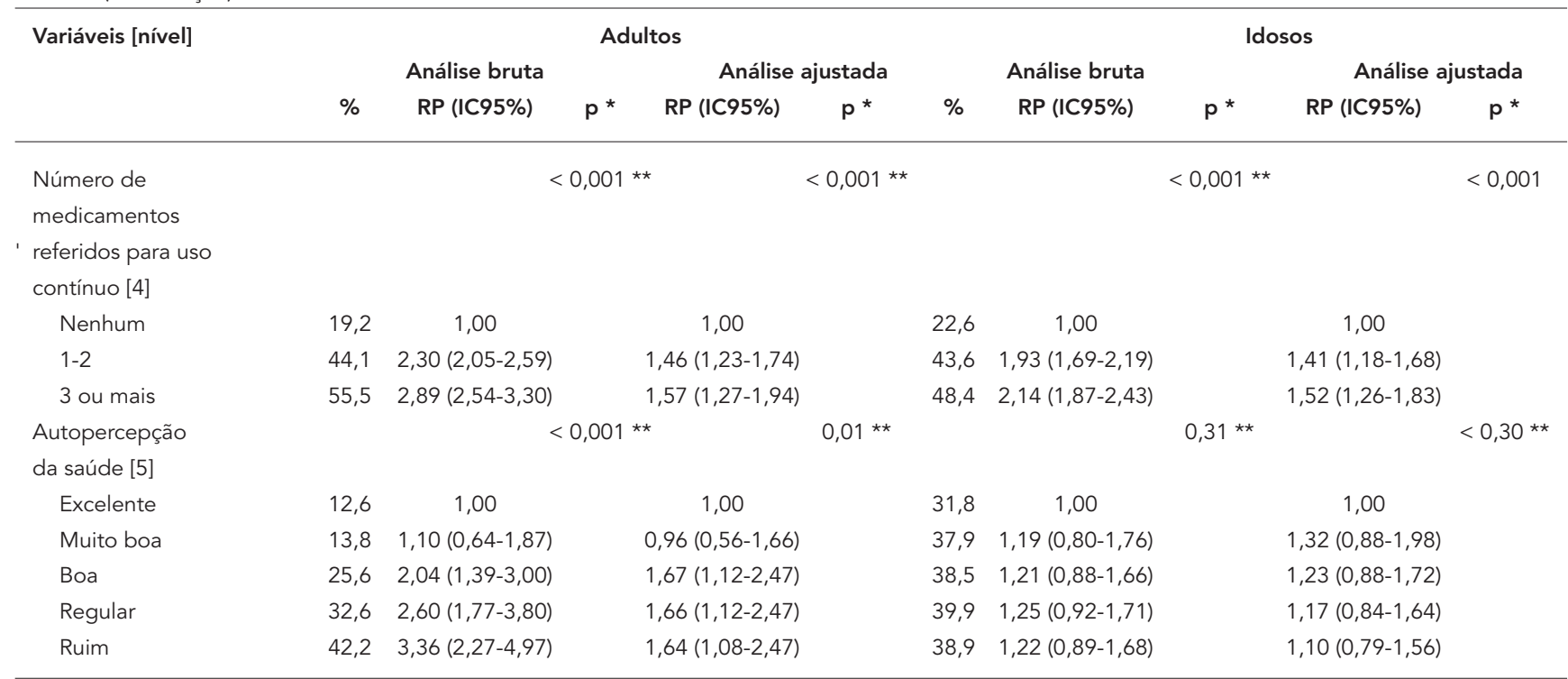

* Valores de p calculados pelo teste de Wald para heterogeneidade;

** Valores de $\mathrm{p}$ calculados pelo teste para tendência linear;

*** De acordo com a classificação da Associação Brasileira de Empresas de Pesquisa (ABEP) 8.

dos a mudar seu comportamento no sentido de aumentarem seus níveis de atividade física. Um estudo realizado na Austrália em 2000, descreveu que $24,2 \%$ dos adultos participantes da pesquisa indicaram ter recebido aconselhamento educativo à atividade física 12 , um valor muito próximo ao observado no presente estudo.

Embora as baixas prevalências de aconselhamento educativo por meio da indicação da atividade física como terapêutica sejam percebidas nos dois modelos de assistência, o modelo do PSF apresenta sempre melhores resultados, e isto está em acordo com os achados de outros estudos, os quais avaliaram a efetividade da atenção básica em relação a possíveis diferenças entre a atuação dos modelos de assistência no Brasil 2,13.

Um achado que vem atender às preocupações atuais em termos de saúde diz respeito a um maior aconselhamento educativo às pessoas idosas. Estudos mostram que o Brasil passa por um período de transição demográfica e epidemiológica 14 , e verifica-se que os resultados aqui apresentados estão de acordo com as estratégias propostas em saúde para esse grupo populacional, pois mostraram que tanto para as prevalências na amostra em geral como nas duas regiões estudadas os idosos sempre foram mais aconselhados sobre a necessidade de se manterem ativos e a realizar atividade física, como uma estratégia de manutenção e prevenção da saúde 15. Esse re- sultado pode ser explicado pela maior exposição, visto que os idosos, por terem mais idade, têm mais tempo de vida e, conseqüentemente, maior possibilidade para receberem aconselhamento. Outra possibilidade seria um maior uso de serviços de saúde, mas estudo recente não encontrou maior freqüência de uso de tais serviços entre os idosos 16 .

Acredita-se que a diferença no aconselhamento educativo à prática de atividade física verificada em favor das mulheres tenha relação com a maior utilização dos serviços de saúde pelas mesmas, principalmente relacionado às causas reprodutivas, mas também, pelo fato de as mulheres avaliarem seu estado de saúde de maneira mais negativa e referirem mais doenças crônicas que os homens, o que já foi relatado em outros estudos 16,17,18. Nossos resultados também são consistentes com a utilização de serviços de saúde em geral no que se refere à idade. Tanto em nosso estudo, referente à orientação para a prática de atividade física, como no estudo de Dias-da-Costa et al. 16, referente à utilização de serviços de saúde em geral, os indivíduos na faixa etária de 50 a 65 anos foram os que apresentaram maior freqüência dos eventos.

A associação encontrada entre aconselhamento educativo à saúde relacionado à atividade física em adultos e idosos e padrão de consumo sócio-econômico está de acordo com o esperado, visto que os indivíduos dos níveis econômicos 
mais altos são os que apresentam menor necessidade em saúde e utilizam com menor freqüência as unidades básicas de saúde ${ }^{19}$. Deve-se considerar que, apesar de a prevalência ainda ser bai$\mathrm{xa}$, algum aconselhamento sobre atividade física chega aos mais pobres, o que pode se traduzir em mudança de comportamento no sentido de um estilo de vida mais saudável nesta população 20,21, mesmo assumindo-se que é um grande desafio promover mudanças de comportamento neste grupo populacional 22.

O estudo de Peterson 23, nos Estados Unidos, mostra que o aumento da prática de aconselhamento para atividade física nos serviços básicos de saúde é um método de promover estilo de vida saudável, e reduz consideravelmente as conseqüências devastadoras para a saúde resultantes de um estilo de vida sedentário. Nosso estudo mostrou que, entre os adultos, o aconselhamento está sendo mais realizado entre os sedentários e, portanto, está de acordo com o que sugere o autor. No entanto, para os idosos, tal associação não foi observada, sugerindo que os profissionais de saúde não investigam o status de atividade física do paciente durante a consulta, pois seria de se esperar que os mais sedentários fossem mais aconselhados a praticarem atividade física.

Nosso estudo também mostra que as pessoas mais necessitadas em relação a agravos à saúde, como hipertensão arterial, diabetes e doença dos nervos, são as que mais receberam aconselhamento para realizar atividade física. Esse achado é positivo, pois existe um amplo corpo de conhecimento mostrando os benefícios da atividade física na prevenção e tratamento de tais morbidades $24,25,26,27,28$.

\section{Resumo}

O estudo descreve a prevalência de aconselhamento educativo à saúde relacionado à atividade física entre as pessoas que utilizaram alguma vez na vida uma unidade básica de saúde, e verifica alguns fatores associados. Estudo com delineamento transversal e amostra aleatória de indivíduos adultos $(N=$ 4.060) e idosos ( $N=4.003)$, moradores em áreas de abrangência de unidades de saúde, em 41 municípios de sete estados das regiões Sul e Nordeste do Brasil. A prevalência de aconselhamento relacionado à atividade física foi de 28,9\% (IC95\%: 27,3-30,4) entre os adultos e de 38,9\% (IC95\%: 37,3-40,6) entre os idosos. A prevalência do desfecho foi sempre maior entre os idosos na região Nordeste, e nos adultos e idosos aten-
Os achados deste trabalho em relação ao maior aconselhamento aos indivíduos que fazem maior utilização de medicamentos e com aqueles que manifestam uma percepção de saúde ruim, nos parecem de acordo com os conhecimentos atuais relacionados à utilização de serviços de saúde. As pessoas que mais consomem medicamentos são as mais necessitadas em termos de saúde, assim como aquelas que pior percebem a sua saúde. Logo, as chances desses indivíduos receberem orientação nas unidades básicas de saúde, ainda que pequenas, são maiores que as dos demais grupos 16 .

Em resumo, a prevalência de aconselhamento educativo à prática de atividade física na unidade básica de saúde, embora já venha ocorrendo, ainda é muito baixa frente às necessidades dos indivíduos. Nossos resultados mostram que é preciso incrementar a participação dos gestores, profissionais e população no sentido de aumentar as prevalências de aconselhamento educativo à população relacionado à prática de atividade física. Deve-se estimular também o aconselhamento em outras áreas, como promoção de alimentação, nutrição, saneamento, planejamento familiar, imunização, tratamento de agravos à saúde, com o objetivo de proporcionar espaços educativos em saúde aos usuários, estimulando a compreensão da importância da mudança de comportamento em direção a um estilo de vida saudável. Por fim, nos parece adequada a participação de profissionais de saúde com conhecimento específico na área da atividade física, entre eles o educador físico, como estratégia de qualificar tanto o grupo profissional da unidade básica de saúde quanto a própria orientação educativa nesta área do conhecimento. didos pelo Programa Saúde da Família. Sexo, padrão de consumo sócio-econômico, tabagismo, sedentarismo, diagnóstico médico de doenças crônicas e uso de medicação contínua também estiveram associados ao desfecho. O aconselhamento à prática de atividade física nas unidades básicas de saúde foi pouco utilizado frente às necessidades dos indivíduos, principalmente em termos de estimular hábitos de vida saudáveis. $\hat{E}$ necessário melhorar a participação dos profissionais das diferentes áreas do conhecimento na condução de aconselhamentos em saúde.

Atividade Motora; Aconselhamento; Adulto; Idoso 


\section{Colaboradores}

F. V. Siqueira colaborou na concepção do artigo, análise de dados, interpretação e redação final do artigo, coordenou o trabalho de campo do estudo, e participou da elaboração dos instrumentos e processamento de dados. M. V. Nahas contribuiu na concepção e revisão do artigo. L. A. Facchini colaborou na revisão do artigo. R. X. Piccini participou da revisão do artigo final. E. Tomasi colaborou na revisão do artigo na fase de concepção em relação ao Projeto de Expansão e Consolidação da Saúde da Família. E. Thumé contribuiu na revisão do artigo e na elaboração das tabelas. D. S. Silveira trabalhou na revisão da análise dos dados, interpretação e revisão final do artigo. P. C. Hallal participou da revisão do trabalho, desde a sua concepção até a redação final na parte específica relacionada à atividade física.

\section{Agradecimentos}

Ao Ministério da Saúde e ao Banco Mundial pelo apoio financeiro.

\section{Referências}

1. Departamento de Gestão da Educação na Saúde, Secretaria de Gestão do Trabalho e da Educação na Saúde, Ministério da Saúde. A educação permanente entra na roda: pólos de educação permanente em saúde: conceitos e caminhos a percorrer. Brasília: Ministério da Saúde; 2005.

2. Facchini LA, Piccini RX, Tomasi E, Thumé E, Silveira DS, Siqueira FV, et al. Desempenho do PSF no Sul e no Nordeste do Brasil: avaliação institucional e epidemiológica da atenção básica à saúde. Ciênc Saúde Coletiva 2006; 11:669-81.

3. Fuscaldo JM. Prescribing physical activity in primary care. WV Med J 2002; 98:250-3.

4. Chakravarthy MV, Joyner MJ, Booth FW. An obligation for primary care physicians to prescribe physical activity to sedentary patients to reduce the risk of chronic health conditions. Mayo Clin Proc 2002; 77:165-73.

5. Börjesson M, Dahlöf B. Physical activity has a key role in hypertension therapy. Lakartidningen 2005; 102:123-4.
6. Facchini LA, Piccini RX, Tomasi E, Thumé E, Teixeira VA, Silveira DS, et al. Avaliação de efetividade da Atenção Básica à Saúde em municípios das regiões Sul e Nordeste do Brasil: contribuições metodológicas. Cad Saúde Pública 2008; 24 Suppl 1:S159-72.

7. Ministério da Saúde. Termo de referência para o estudo de linha de base nos municípios selecionados para o componente 1 do PROESF. Brasília: Ministério da Saúde; 2004.

8. Associação Brasileira de Empresas de Pesquisa. Critério de classificação econômica do Brasil. São Paulo: Associação Brasileira de Empresas de Pesquisa; 2002.

9. Craig CL, Marshall AL, Sjostrom M, Bauman AE, Booth ML, Ainsworth BE, et al. International physical activity questionnaire: 12-country reliability and validity. Med Sci Sports Exerc 2003; 35: 1381-95.

10. Barros AJ, Hirakata VN. Alternatives for logistic regression in cross-sectional studies: an empirical comparison of models that directly estimate the prevalence ratio. BMC Med Res Methodol 2003; $3: 21$. 
11. Eden KB, Orleans CT, Mulrow CD, Pender NJ, Teutsch SM. Does counseling by clinicians improve physical activity? A summary of the evidence for the U.S. Preventive Services Task Force. Ann Intern Med 2002; 137:208-15.

12. Eakin E, Brown W, Schofield G, Mummery K, Reeves M. General practitioner advice on physical activity: who gets it? Am J Health Promot 2007; 21:225-8.

13. Siqueira FV, Facchini LA, Hallal PC. The burden of fractures in Brazil: a population-based study. Bone 2005; 37:261-6.

14. Monteiro CA. Novos e velhos males da saúde no Brasil: a evolução do país e de suas doenças. São Paulo: Editora Hucitec/Universidade de São Paulo; 1995.

15. Hirvensalo M, Heikkinen E, Lintunen T, Rantanen T. The effect of advice by health care professionals on increasing physical activity of older people. Scand J Med Sci Sports 2003; 13:231-6.

16. Dias-da-Costa JS, Facchini LA.Use of outpatient services in an urban area of Southern Brazil: place and frequency. Rev Saúde Pública 1997; 31:360-9.

17. Dias-da-Costa JS, Olinto MTA, Gigante DP, Menezes AMB, Macedo S, Daltoé T, et al. Utilização de serviços ambulatoriais de saúde em Pelotas, Rio Grande do Sul, Brasil: alguns fatores relacionados com as consultas médicas acima da média. Cad Saúde Pública 2008; 24:353-63.

18. Travassos C, Viacava F, Pinheiro R, Brito A. Utilização dos serviços de saúde no Brasil: gênero, características familiares e condição social. Rev Panam Salud Pública 2002; 11:356-73.
19. Macinko JA, Starfield B. Annotated bibliography on equity in health, 1980-2001. Int J Equity Health 2002; $1: 1$.

20. Ammouri AA, Neuberger G, Nashwan AJ, Al-Haj AM. Determinants of self-reported physical activity among Jordanian adults. J Nurs Scholarsh 2007; 39:342-8.

21. Märki A, Bauer GB, Angst F, Nigg CR, Gillmann G, Gehring TM. Systematic counselling by general practitioners for promoting physical activity in elderly patients: a feasibility study. Swiss Med Wkly 2006; 136:482-8.

22. Drevenhorn E, Kjellgren K, Bengtson A. Outcomes following a programme for lifestyle changes with people with hypertension. J Clin Nurs 2007; 16: 144-51.

23. Peterson JA. Get moving! Physical activity counseling in primary care. J Am Acad Nurse Pract 2007; 19:349-57.

24. Shepard RJ. Physical activity, fitness and health: the current consensus. Quest 1995; 47:228-303.

25. Zhao G, Ford ES, Li C, Mokdad AH. Are United States adults with coronary heart disease meeting physical activity recommendations? Am J Cardiol 2008; 101:557-61.

26. Löllgen H, Völker K, Böckenhoff A, Löllgen D. Physical activity and prevention of cardiovascular diseases. Herz 2006; 31:519-23.

27. Constantini N, Harman-Boehm I, Dubnov G. Exercise prescription for diabetics: more than a general recommendation. Harefuah 2005; 144:717-23.

28. Paluska SA, Schwenk TL. Physical activity and mental health: current concepts. Sports Med 2000; 29:167-80.

Recebido em 09/Abr/2008

Versão final reapresentada em 26/Mai/2008

Aprovado em 06/Jun/2008 